\title{
Human phosphoglycerate dehydrogenase produces the oncometabolite D-2-hydroxyglutarate and promotes histone methylation
}

\author{
Jing Fan ${ }^{1}$, Xin Teng ${ }^{1 *}$, Ling Liu', Ryan Looper ${ }^{2}$, Joshua Rabinowitz ${ }^{1,3}$ \\ From Metabolism, Diet and Disease 2014: Cancer and metabolism \\ Washington DC, USA. 28-30 May 2014
}

\section{Background}

Human phosphoglycerate dehydrogenase (PHGDH), the first enzyme in the serine biosynthetic pathway, is genomically amplified in various tumors [1-3]. In such cells, PHGDH knockdown is not fully rescued by exogenous serine [2], suggesting possible additional roles for the enzyme.

\section{Materials and methods}

To analyze the catalytic activity of PHGDH on possible alternative substrates, we conducted in vitro biochemical assays on recombinant human $\mathrm{PHGDH}$. The reaction rate in the reductive direction with various substrates was monitored based on NADH consumption, measured by absorbance at $340 \mathrm{~nm}$. The identity and chirality of products were analyzed by liquid chromatography-mass spectrometry (LC-MS) and by gas chromatography-mass spectrometry (GC-MS) after (R)-2-butanol derivatization. To analyze the intracellular function of PHGDH, we generated stable PHGDH knockdown cell lines, using two shRNA sequences. Metabolite levels in the knockdown cells were analyzed by LC-MS [4], and DNA and histone methylation was analyzed by immunoblotting.

\section{Results}

Here we show that, in addition to catalyzing oxidation of 3-phosphoglycerate, PHGDH catalyzes NADH-dependent reduction of $\alpha$-ketoglutarate to the oncometabolite D-2-hydroxyglutarate (D-2HG) and promotes histone methylation. The impact of PHGDH knockdown was studied in three cell lines with amplified PHGDH: MDAMB-468, BT-20, and HCC70. Knockdown of PHGDH decreased 2HG levels in MDA-MB-468 and BT-20 cells, but not HCC70 cells (which had the highest 2HG levels of the three tested cell lines, with levels in MDA-MB-468 and BT-20 cells far below those found in cells with mutant IDH). These results suggest that PHGDH contributes to physiological 2HG pools, but may not produce high enough concentrations to cause pathology. Interestingly, in all three cell lines, PHGDH knockdown substantially decreased histone methylation. The mechanism underlying the decreased methylation upon PHGDH knockdown remains unclear, but it could be restored in MDA-MB-468 and BT-20 cells by addition of D-2HG ester.

\section{Conclusion}

PHGDH may promote histone methylation by mechanisms including 2-HG production.

\section{Authors' details \\ 'Lewis-Sigler Institute for Integrative Genomics and Department of Chemistry, Princeton University, Princeton, NJ, USA. ${ }^{2}$ Department of Chemistry, University of Utah, Salt Lake City, UT, USA. ${ }^{3}$ Rutgers Cancer Institute of New Jersey, New Brunswick, NJ, USA.}

Published: 28 May 2014

\section{References}

1. Mullarky E, Mattaini KR, Vander Heiden MG, Cantley LC, Locasale JW: PHGDH amplification and altered glucose metabolism in human melanoma. Pigment Cell Melanoma Res 2011, 24:1112-5.

2. Possemato $R$, et al: Functional genomics reveal that the serine synthesis pathway is essential in breast cancer. Nature 2011, 476:346-50.

3. Locasale JW, et al: Phosphoglycerate dehydrogenase diverts glycolytic flux and contributes to oncogenesis. Nat Genet 2011, 43:869-874.

4. Lu W, et al: Metabolomic analysis via reversed-phase ion-pairing liquid chromatography coupled to a stand alone orbitrap mass spectrometer. Anal Chem 2010, 82:3212-3221. 
doi:10.1186/2049-3002-2-S1-P75

Cite this article as: Fan et al: Human phosphoglycerate dehydrogenase

produces the oncometabolite D-2-hydroxyglutarate and promotes histone methylation. Cancer \& Metabolism 2014 2(Suppl 1):P75.

Submit your next manuscript to BioMed Central and take full advantage of:

- Convenient online submission

- Thorough peer review

- No space constraints or color figure charges

- Immediate publication on acceptance

- Inclusion in PubMed, CAS, Scopus and Google Scholar

- Research which is freely available for redistribution

Submit your manuscript at www.biomedcentral.com/submit

() BioMed Central 